\title{
A falta de dentição funcional está associada ao comprometimento das funções bucais entre adultos brasileiros
}

\author{
The absence of functional dentition is associated with the lack \\ of commitment to oral functions among Brazilian adults
}

João Gabriel Silva Souza ${ }^{1}$

Vinicius Aguiar Lages ${ }^{1}$

Aline Araujo Sampaio ${ }^{1}$

Tamirys Caroline Silva Souza ${ }^{2}$

Andrea Maria Eleutério de Barros Lima Martins ${ }^{3}$

${ }^{1}$ Faculdade de Odontologia de Piracicaba, Universidade Estadual de Campinas. Av. Limeira 901, Areião. 13414018 Piracicaba SP Brasil. jgabriel.ssouza@

yahoo.com.br

${ }^{2}$ Faculdades Unidas do

Norte de Minas. Montes

Claros MG Brasil.

${ }^{3}$ Departamento de

Odontologia, Universidade

Estadual de Montes Claros.

\begin{abstract}
The scope of this study was to identify if the absence of functional dentition (FD) is associated with a lack of commitment to oral functions/ daily activities among Brazilian adults. For this purpose, data from the National Survey of Oral Health (referred to as SB Brasil 2010) was used. FD was evaluated by the criterion proposed by the WHO (at least 20 teeth in the mouth). The Oral Impacts on Daily Performance (OIDP) questionnaire was used to evaluate daily activities/oral functions. Descriptive, bivariate (chisquared) and multiple (logistic regression) analysis was conducted, and the odds ratio estimated with a 95\% confidence interval (OR/CI95\%). The research evaluated 9,564 adults, of which 2,200 adults (20.5\%) were considered to have poor FD, and at least one of the daily activities/ oral functions evaluated had an impact among $55 \%$ of adults. The lack of FD between adults was associated $(p \leq 0.05)$ with the impact on speech (1.88/1.33-2.64) and being ashamed to smile or talk (1.35/1.00-1.83). A considerable prevalence of lack of FD was identified, this absence being associated with the lack of commitment to the daily activities/oral functions. Dental rehabilitation of patients with a lack of FD should consider the restoration of these lost oral functions (speech and being ashamed to smile or talk).
\end{abstract}

Key words Functional dentition, Tooth loss, Oral health, Speech, Adults
Resumo Objetivou-se identificar se a falta de dentição funcional (DF) está associada com o comprometimento das funções bucais/atividades diárias entre adultos brasileiros. Para isto, foram utilizados dados da Pesquisa Nacional de Saúde Bucal - SB Brasil 2010. A DF foi avaliada pelo critério proposto pela Organização Mundial de Saúde (pelo menos 20 dentes na boca). O instrumento Impactos Odontológicos nos Desempenhos Diários (IODD) foi utilizado para avaliar as atividades diárias/funções bucais. Análises descritivas, bivariadas (Qui-quadrado) e múltiplas (Regressão Logística) foram realizadas, sendo estimado o odds ratio e o intervalo de confiança 95\% (OR/IC95\%). Foram incluidos e avaliados 9564 adultos. Foram considerados sem DF 2200 adultos (20,5\%). Tiveram impacto em pelo menos uma das atividades diárias/funções bucais avaliadas, 55,0\% dos adultos. A falta de DF entre adultos foi associada $(p \leq 0,05)$ com o impacto na fala $(1,88 / 1,33-2,64)$ e vergonha ao sorrir ou falar (1,35/1,00-1,83). Uma prevalência considerável de falta de DF foi identificada, esta ausência foi associada às atividades diárias/funções bucais (fala e vergonha ao sorrir e falar). A reabilitação dentária de pacientes sem DF deve considerar a devolução destas funções bucais perdidas (fala e vergonha ao sorrir ou falar).

Palavras-chave Dentição funcional, Perda dentária, Saúde bucal, Fala, Adulto 


\section{Introdução}

A perda dentária tem apresentado altas taxas de prevalência em diferentes partes do mundo, como demonstrado por revisão sistemática da literatura ${ }^{1}$. Sua ocorrência pode comprometer a execução das funções bucais². Pessoas com ocorrência de perdas dentárias tem 2,7 vezes mais chance de relatar dificuldades relacionadas a mastigação, quando comparadas às sem perdas ${ }^{3}$. Esta perda tem comprometido também a acuidade auditiva ${ }^{4}$, a capacidade de comunicação ${ }^{2}$ e a interação social ${ }^{5}$, podendo impactar negativamente na qualidade de vida relacionada à saúde bucal dos indivíduos ${ }^{6}$. Ressalta-se que, para uma adequada e satisfatória execução das funções bucais, é necessário a presença de uma dentição funcional.

A dentição funcional (DF) tem sido definida pela Organização Mundial de Saúde (OMS) como a presença/retenção de uma dentição natural, com estética, que seja funcional, e com a presença de pelo menos 20 dentes ao longo da vida, sem a necessidade de reabilitação protéti$\mathrm{ca}^{7}$. Este tipo de dentição tem sido caracterizado e determinado por diferentes métodos de avaliação ${ }^{8}$. Dentre eles, o conceito e critério da OMS tem sido amplamente utilizado na literatura, sendo aplicado em estudos populacionais em adultos $^{8-10}$ e idosos ${ }^{11}$. O uso deste critério nestas populações tem apontando prevalência superior a 70\% de indivíduos com dentição funcional ( $\geq$ 20 dentes $)^{8-11}$. A presença de DF tem sido associada a indivíduos de classe social mais alta ${ }^{10}$, maior ingestão de nutrientes na $\operatorname{dieta}^{12}$, com menor necessidade de reabilitação protética ${ }^{13} \mathrm{e}$ com melhores comportamentos de saúde bucal ${ }^{9}$. Portanto, a presença de DF parece indicar melhores condições de saúde bucal e, talvez, facilitar a execução das funções bucais e atividades diárias dos indivíduos.

No entanto, não tem sido bem esclarecido na literatura se a falta de DF está associada ao comprometimento da execução de atividades diárias/ funções bucais. Considerando as melhorias ocasionadas pela presença de DF e a perda de funções bucais decorrente da perda dentária, tem-se por hipótese que a falta de DF está associada ao comprometimento da execução de atividades diárias/funções bucais. Portanto, o presente estudo tem por objetivo identificar se a falta de DF está associada ao comprometimento de atividades diárias/funções bucais.

\section{Metodologia}

\section{Delineamento e amostragem}

Trata-se de um estudo transversal que utilizou dados extraídos da Pesquisa Nacional de Saúde Bucal da População Brasileira, conduzida em 2010 (SB Brasil 2010) de acordo com os critérios propostos pela $\mathrm{OMS}^{14}$. Uma amostra representativa da população brasileira, em faixas etárias e idades índices, foi entrevistada e examinada em seus domicílios sobre suas características sociodemográficas e condições de saúde bucal.

Utilizou-se uma amostragem probabilística por conglomerados, em múltiplos estágios, com probabilidade proporcional ao tamanho e considerando um efeito de desenho (deff) igual a 2. Cada capital das unidades federativas mais o Distrito Federal e uma amostra de municípios do interior de cada macrorregião brasileira foram consideradas domínios da pesquisa. A seleção amostral foi estruturada em dois estágios para as capitais e Distrito Federal e em três estágios para os municípios do interior das cinco macrorregiões brasileiras, sendo as unidades amostrais sorteadas pela técnica de Probabilidade Proporcional ao Tamanho ${ }^{14,15}$.

Os exames e entrevistas foram realizados por cirurgiões-dentistas previamente treinados e calibrados pela técnica do consenso, sendo o valor mínimo aceitável de kappa para cada examinador, grupo etário e agravo estudado igual a $0,65^{14,15}$. Na presente investigação utilizou-se um recorte do banco de dados e foi incluída nas análises a população adulta ( 35 a 44 anos) para avaliação de perda dentária, que foi utilizada para estimar a presença de dentição funcional.

\section{Dentição funcional}

A variável dependente - falta/ausência de dentição funcional - foi avaliada a partir da definição proposta pela OMS sendo preconizado a presença de 20 dentes ou mais para o indivíduo ser considerado com dentição funcional ${ }^{7}$. A construção da variável dependente foi realizada a partir do componente " $\mathrm{P}$ - perdido", que representa o número de dentes perdidos por cárie no índice CPO-D. Este índice é o somatório do número de dentes que apresentam lesões cariosas (componente $\mathrm{C}$ ), dentes perdidos (componente $\mathrm{P}$ ) e restaurados (componente $\mathrm{O}$ ), e usado para avaliar a experiência presente e passada do ataque da cárie dental à dentição permanente. Dessa forma, considerando os 32 dentes presentes nas 
arcadas dentárias, os indivíduos que perderam no máximo 12 dentes (ou seja, tem no mínimo 20 dentes na boca) foram considerados "com dentição funcional"; os que perderam mais de 12 dentes (ou seja, menos de 20 dentes na boca) foram considerados "sem dentição funcional". A partir da variável dependente dicotomizada, foi caracterizado a falta de dentição funcional (menos de 20 dentes na boca).

\section{Impactos nas atividades de vida diária}

O impacto da falta de dentição funcional na vida diária dos adultos brasileiros foi avaliado pelo instrumento Impactos Odontológicos nos Desempenhos Diários (IODD). O instrumento é composto por nove atividades da vida diária, sendo elas: dificuldade para comer, incômodo para escovar, nervosismo ou irritação, vergonha ao sorrir, atrapalhar o sono, influência no lazer, dificuldade em falar, atrapalhar o estudo/trabalho e influência no esporte. Cada item foi precedido da pergunta Algumas pessoas têm problemas que podem ter sido causados pelos dentes. Das situações abaixo, quais se aplicam a (o) sr(a), nos últimos seis meses?, sendo as opções de resposta: não, sim e não sabe ou não quis responder (considerado informação faltante $)^{16}$. Para avaliação do impacto na vida diária foi construída uma variável dicotômica pela junção dos nove itens, sendo as opções de resposta: "Sem impacto" (impacto em nenhum dos itens) e "Com impacto" (impacto em pelo menos um item). Além disso, considerou-se como variáveis independentes cada um dos nove itens (perguntas) do IODD, com intuito de verificar qual atividade diária específica poderia estar associada com a falta de dentição funcional. Estes também foram dicotomizados (sem impacto/ Com impacto).

\section{Variáveis independentes \\ - sociodemográficas}

Para controle dos fatores associados por características sociodemográficas, incluiu-se também como variáveis independentes: sexo (Masculino/Feminino), cor da pele (Branco/Negro, pardo, amarelo, indígena) e renda familiar (Mais de 500 reais/Até 500 reais).

\section{Análise estatística e considerações éticas}

$\mathrm{Na}$ análise dos dados, empregou-se o software SPSS ${ }^{\circledR}$ Statistics 18.0. Como o estudo envolveu amostra complexa por conglomerados, foi feita a correção pelo efeito do desenho amostral, levando em consideração o efeito dos conglomerados e a atribuição de ponderações aos elementos amostrados. Para variáveis categóricas, a análise descritiva incluiu a distribuição da amostra e frequência relativa corrigida (\%). Para as variáveis quantitativas, foram estimadas a média e o intervalo de confiança de 95\%, também corrigidos pelo efeito do desenho amostral. Para verificar a associação da falta de dentição funcional com as atividades de vida diária, foram realizadas análises bivariada e múltipla. $\mathrm{Na}$ análise bivariada foram estimados odds ratio (OR) e intervalos de confiança de 95\% (IC95\%), com a correção pelo efeito do desenho amostral (OR/IC95\%). As variáveis independentes que apresentaram nível de significância menor ou igual a 20\% (p < 0,20) foram selecionadas para a análise múltipla. A análise múltipla conduzida foi a regressão logística com modelo ajustado em nível de significância de $5 \%(\mathrm{p} \leq 0,05)$ para as variáveis que permaneceram associadas ao desfecho (falta de dentição funcional).

O levantamento epidemiológico foi conduzido respeitando os princípios éticos da Resolução do Conselho Nacional de Saúde nº196/96, sendo aprovado e registrado pela Comissão Nacional de Ética em Pesquisa (CONEP).

\section{Resultados}

9564 adultos foram incluídos e avaliados quanto a DF. A média de idade da amostra foi de 39,3 anos (IC95\% 39,2-39,5). A partir do critério da OMS, 2200 (20,5\%) adultos não possuíam DF, sendo 7364 (79,5\%) considerados com DF. Identificou-se que a maioria da amostra era composta por mulheres e de raça negra/amarelo/pardo/ indígena. Quanto ao impacto das condições de saúde bucal na vida diária, 55,0\% dos adultos possuíam impacto em pelo menos uma das atividades diárias (Tabela 1 ).

$\mathrm{Na}$ análise bivariada, dentre as atividades diárias identificou-se que apenas o impacto na escovação e na prática de esportes não foram associados $(\mathrm{p} \geq 0,20)$ com a falta de DF (Tabela 1$)$.

Dentre as atividades de vida diária/funções bucais avaliadas, identificou-se que entre os adultos sem dentição funcional, as atividades com maior prevalência de impacto foram a fala e vergonha ao sorrir ou falar (Figura 1).

$\mathrm{Na}$ análise múltipla, identificou-se que a falta de dentição funcional foi associada ao impacto na fala $(\mathrm{p}<0,001)$ e na vergonha em sorrir ou 
Tabela 1. Análise descritiva e bivariada das variáveis independentes em relação à falta de dentição funcional entre adultos brasileiros (35-44 anos) (2010). $\mathrm{n}=9564$.

\begin{tabular}{|c|c|c|c|c|c|}
\hline \multirow{2}{*}{$\begin{array}{l}\text { Variáveis } \\
\text { Dependente }\end{array}$} & \multirow[b]{2}{*}{$\mathbf{N}$} & \multirow[b]{2}{*}{$\%$} & \multicolumn{3}{|c|}{$\begin{array}{l}\text { Falta de dentição } \\
\text { funcional }\end{array}$} \\
\hline & & & $\%$ & OR & p \\
\hline \multicolumn{6}{|l|}{ Dentição funcional } \\
\hline Sim & 7364 & 79,5 & & & \\
\hline Não & 2200 & 20,5 & - & - & - \\
\hline \multicolumn{6}{|l|}{ Características Socioeconômicas } \\
\hline \multicolumn{6}{|l|}{ Sexo } \\
\hline Masculino & 3277 & 37,7 & 16,5 & 1 & \\
\hline Feminino & 6287 & 62,3 & 22,9 & 1,50 & 0,001 \\
\hline \multicolumn{6}{|l|}{ Cor da pele } \\
\hline Branco & 4049 & 47,6 & 17,9 & 1 & \\
\hline $\begin{array}{l}\text { Negro/ Amarelo/ Pardo/ } \\
\text { Indígena }\end{array}$ & 5515 & 52,4 & 22,8 & 1,35 & 0,011 \\
\hline \multicolumn{6}{|l|}{ Renda familiar } \\
\hline Mais de 500 reais & 7933 & 86,2 & 19,1 & 1 & \\
\hline Até 500 reais & 1404 & 13,8 & 29,5 & 1,77 & $<0,001$ \\
\hline \multicolumn{6}{|l|}{ Impacto nas atividades diárias } \\
\hline \multicolumn{6}{|l|}{ IODD geral } \\
\hline Sem impacto & 4562 & 45,0 & 17,5 & 1 & \\
\hline Com impacto & 5002 & 55,0 & 22,9 & 1,40 & 0,003 \\
\hline \multicolumn{6}{|l|}{ IODD1 - Mastigação/comer* } \\
\hline Sem impacto & 6545 & 67,9 & 18,5 & 1 & \\
\hline Com impacto & 2979 & 32,1 & 24,3 & 1,40 & 0,007 \\
\hline \multicolumn{6}{|l|}{ IODD2 - Escovação* } \\
\hline Sem impacto & 7260 & 73,8 & 20,6 & 1 & \\
\hline Com impacto & 2271 & 26,2 & 20,0 & 0,96 & 0,805 \\
\hline \multicolumn{6}{|l|}{$\begin{array}{l}\text { IODD3 - Nervosismo/ } \\
\text { Irritação* }\end{array}$} \\
\hline Sem impacto & 7498 & 75,9 & 19,5 & 1 & \\
\hline Com impacto & 2023 & 24,1 & 23,3 & 1,25 & 0,057 \\
\hline \multicolumn{6}{|l|}{ IODD4 - Lazer* } \\
\hline Sem impacto & 8224 & 85,4 & 19,6 & 1 & \\
\hline Com impacto & 1309 & 14,6 & 25,2 & 1,38 & 0,050 \\
\hline \multicolumn{6}{|l|}{ IODD5 - Praticar esportes ${ }^{\star}$} \\
\hline Sem impacto & 8943 & 93,9 & 20,3 & 1 & \\
\hline Com impacto & 564 & 6,1 & 22,7 & 1,15 & 0,575 \\
\hline \multicolumn{6}{|l|}{ IODD6 - Fala* } \\
\hline Sem impacto & 8275 & 86,7 & 18,3 & 1 & \\
\hline Com impacto & 1265 & 13,3 & 34,3 & 2,33 & $<0,001$ \\
\hline \multicolumn{6}{|l|}{$\begin{array}{l}\text { IODD7 - Vergonha para } \\
\text { sorrir ou falar* }\end{array}$} \\
\hline Sem impacto & 7087 & 73,7 & 17,5 & 1 & \\
\hline Com impacto & 2443 & 26,3 & 28,8 & 1,90 & $<0,001$ \\
\hline \multicolumn{6}{|l|}{ IODD8 - Estudar/Trabalhar* } \\
\hline Sem impacto & 8522 & 89,4 & 19,9 & 1 & \\
\hline Com impacto & 1003 & 10,6 & 24,8 & 1,32 & 0,143 \\
\hline \multicolumn{6}{|l|}{ IODD9 - Sono/Dormir* } \\
\hline Sem impacto & 8038 & 82,7 & 20,5 & 1 & \\
\hline Com impacto & 1478 & 17,3 & 20,2 & 0,97 & 0,861 \\
\hline
\end{tabular}

* variação no n por perda de informação. OR - Odds ratio. IODD - Impactos Odontológicos nos Desempenhos Diários. falar $(p=0,050)$ pelos adultos. Em adição, o desfecho (falta de dentição funcional) foi associado $(\mathrm{p} \leq 0,05)$ ao sexo e renda familiar (Tabela 2).

\section{Discussão}

Uma parcela considerável da população adulta brasileira não tem $\mathrm{DF}$ (20,5\%), de acordo com o critério proposto pela OMS. A falta de DF esteve associada ao comprometimento da fala, resultado identificado a partir da associação com impacto na fala e na vergonha de sorrir ou falar. Portanto, a ausência de DF parece comprometer a função bucal e impactar negativamente na vida diária dos indivíduos.

Ao considerar a prevalência de perdas dentárias entre adultos brasileiros ${ }^{17}$, esperava-se uma maior prevalência da ausência de DF, sendo esta situação presente em aproximadamente $1 / 4$ da população. Ressalta-se que, a dentição funcional foi avaliada conforme critério proposto pela OMS (pelo menos 20 dentes na boca) ${ }^{7}$. No entanto, diferentes índices têm sido propostos recentemente e utilizados na literatura ${ }^{18}$, enfatizando a necessidade de se considerar também a distribuição dos dentes na arcada e a condição periodontal. Estudos populacionais realizados em amostra de representativa de adultos brasileiros, ou de municípios específicos, como no caso de Belo Horizonte, tem apontando prevalência semelhante de falta de dentição funcional ${ }^{8,9}$. Prevalência inferior tem sido descrita em adultos ingleses $(\approx 15 \%)^{10}$. Destaca-se ainda, que em lugares como os Estados Unidos, os idosos, uma faixa etária altamente acometida por perda dentaria, tem apresentado prevalência inferior de falta de dentição funcional $^{11}$. A prevalência de falta de DF entre adultos merece atenção, já que a perda dentária grave aumenta com o avançar da idade, atingindo um pico aos 65 anos$^{1}$. Além disso, a OMS, a Federação Dental Internacional e a Associação Internacional de pesquisa Odontológica propõem como objetivo para o ano de 2020 que haja uma redução na perda dentária, principalmente entre adultos, assim como uma redução do número de indivíduos com dificuldades para executar as funções bucais ${ }^{19}$. Com intuito de alcançar tais objetivos, a população adulta deve ser investigada a fim de preservar os elementos dentais em idades futuras. Apesar da DF ser caracterizada por quantidade de elementos dentais suficiente para uma favorável estética e ser funcional, sua presença tem sido aliada a reabilitação dentária ${ }^{11}$. Os impactos das perdas dentárias na execução de funções bucais, 


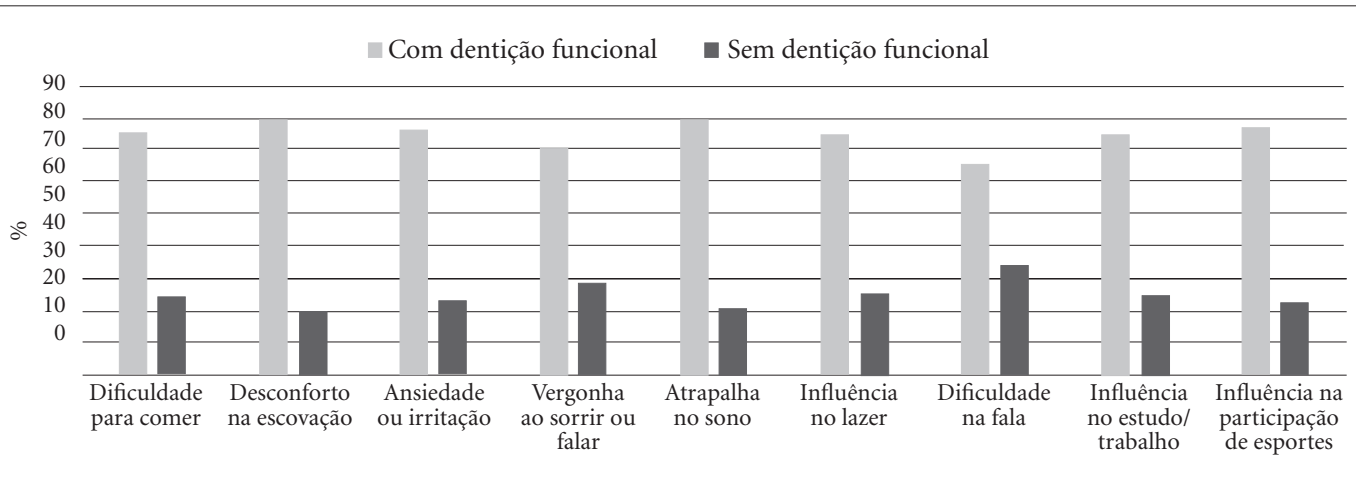

Figura 1. Prevalência do impacto nas atividades diárias/funções bucais entre adultos brasileiros com e sem dentição funcional (2010).

tais como a mastigação, tem sido descritos na literatura ${ }^{3}$. Portanto, era esperado que a ausência de DF fosse associada ao comprometimento de funções bucais e atividades diárias.

A falta de DF foi associada ao comprometimento da fala e com a vergonha de sorrir ou falar devido as condições de saúde bucal. A presença de dentes é essencial para pronúncia de certas consoantes $^{20}$. Nesse sentido, a perda dentária pode alterar a performance da fala ${ }^{21}$. Portanto, uma maior quantidade de dentes perdidos e, consequentemente, falta de DF afeta a fala, explicando a associação identificada. Ressalta-se também que a perda dentária pode alterar a dimensão vertical de oclusão e o espaço funcional livre da fala, além de levar a desordens articulares, o que prejudicam a fala ${ }^{6,22}$. Apesar de indivíduos com DF apresentarem também reabilitação dentária $^{11}$, esta deve ser realizada com cautela. Evidências clínicas tem apontado que dependendo do protocolo de tratamento utilizado a reabilitação de elementos perdidos pode intervir na fala ${ }^{22-24}$. Jacobs et al. ${ }^{23}$ identificaram que a reabilitação dentária com próteses implanto-suportadas leva a uma maior chance de desordens fonéticas, quando comparados aos indivíduos com dentição natural. Pacientes sem DF devem ser reabilitados com intuito de devolver suas funções bucais, inclusive o conforto na fala.

Considerando que a perda dentária pode comprometer as funções bucais ${ }^{2}$ e a qualidade de vida ${ }^{6}$, é de se esperar uma relação entre a falta de DF com a vergonha de sorrir ou falar. Além disso, os distúrbios relacionados a fala podem comprometer o processo de comunicação, o que pode estar ligado a vergonha em falar.
Tabela 2. Análise múltipla das variáveis independentes associadas à falta de dentição funcional entre adultos brasileiros (2010).

\begin{tabular}{lrrc}
\hline \multicolumn{1}{c}{ Variáveis } & OR & IC 95\% & p \\
\hline Característccas & & & \\
Socioeconômicas & & & \\
$\quad$ Sexo & & & \\
$\quad$ Masculino & 1,44 & $1,14-1,82$ & 0,002 \\
$\quad$ Feminino & & & \\
Renda familiar & 1 & & \\
$\quad$ Mais de 500 reais & 1,63 & $1,26-2,12$ & $<0,001$ \\
$\quad$ Até 500 reais & & & \\
Impacto nas atividades diárias & & & \\
$\quad$ IODD6 - Fala & 1 & & \\
$\quad$ Sem impacto & 1,88 & $1,33-2,64$ & $<0,001$ \\
$\quad$ Com impacto & & & \\
IODD7 - Vergonha para & & & \\
$\quad$ sorrir ou falar & 1 & & \\
$\quad$ Sem impacto & 1,35 & $1,00-1,83$ & 0,050 \\
$\quad$ Com impacto &
\end{tabular}

OR - Odds ratio. IC95\% - Intervalo de confiança 95\%.

Apesar dos resultados identificarem que apenas a fala e a vergonha ao falar ou sorrir foi associada à falta de DF, esperava-se que o comprometimento de outras funções bucais/atividades diárias também fossem associadas. Por exemplo, estudos clínicos têm demonstrado o efeito da perda dentária no comprometimento da mastigação ${ }^{3,25}$. O impacto das funções bucais/atividades diárias foi aferido no presente estudo pela percepção do próprio indivíduo, ou seja, uma 
medida subjetiva. A análise subjetiva permite identificar o quanto o indivíduo se sente afetado pela condição bucal ${ }^{26}$, o que pode proporcionar a busca pelo tratamento odontológico ${ }^{27}$.

Apesar das variáveis sociodemográficas terem sido utilizadas para controle dos fatores associados, identificou-se um perfil de desigualdade social na falta de DF. Maior prevalência de falta de DF foi identificada entre mulheres e adultos com menor renda familiar. Desigualdades sociais na ocorrência de problemas bucais têm sido identi- ficadas no Brasil ${ }^{17,28}$, inclusive relacionadas à perda dentária ${ }^{17}$. Este tipo de perfil de desigualdade pode ser decorrente de iniquidades relacionados ao acesso aos serviços odontológicos, o que pode incrementar a prevalência de problemas bucais ${ }^{29}$.

Conclui-se que uma parcela considerável dos adultos estudados não tem $\mathrm{DF}$, sendo esta ausência associada ao comprometimento na fala e na vergonha ao sorrir ou falar. Sugere-se que a falta de DF esteja relacionada a um comprometimento na comunicação dos indivíduos acometidos.

\section{Colaboradores}

JGS Souza e AMEBL Martins participaram da organização e análise dos dados, redação do artigo e revisão crítica. VA Lages, AA Sampaio e TCS Souza participaram da redação do artigo. 


\section{Referências}

1. Kassebaum NJ, Bernabé E, Dahiya M, Bhandari B, Murray CJ, Marcenes W. Global Burden of Severe Tooth Loss: A Systematic Review and Meta-analysis. J Dent Res 2014; 93(Supl. 7):20S-28S.

2. Gotfredsen K, Walls AW. What dentition assures oral function? Clin Oral Implants Res 2007; 18(Supl. 3):3445.

3. Gilbert GH, Meng X, Duncan RP, Shelton BJ. Incidence of tooth loss and prosthodontic dental care: effect on chewing difficulty onset, a component of oral health-related quality of life. J Am Geriatr Soc 2004; 52(6):880-885.

4. Lawrence HP, Garcia RI, Essick GK, Hawkins R, Krall EA, Spiro A, Vokonas PS, Kong L, King T, Koch GG. A longitudinal study of the association between tooth loss and age-related hearing loss. Spec Care Dentist 2001; 21(4):129-140.

5. Saintrain MV, Souza EH. Impact of tooth loss on the quality of life. Gerodontology 2012; 29(2):e632-636.

6. Gerritsen AE, Allen PF, Witter DJ, Bronkhorst EM, Creugers NH. Tooth loss and oral health-related quality of life: a systematic review and meta-analysis. Health Qual Life Outcomes 2010; 8:126.

7. World Health Organization (WHO). Recent advances in oral health: WHO Technical Report Series. Geneva: WHO; 1992.

8. Chalub LL, Martins CC, Ferreira RC, Vargas AM. Functional Dentition in Brazilian Adults: An Investigation of Social Determinants of Health (SDH) Using a Multilevel Approach. PLoS ONE 2016; 11(2):e0148859.

9. Chalub LLFH, Borges CM, Ferreira RC, Haddad JPA, Ferreira EF, Vargas AMD. Association between social determinants of health and functional dentition in 35-year-old to 44-year-old Brazilian adults: a population-based analytical study. Community Dent Oral Epidemiol 2014; 42(6):503-516.

10. Bernabé E, Sheiham A. Tooth Loss in the United Kingdom - Trends in Social Inequalities: An Age-Period-and-Cohort Analysis. PLoS ONE 2014; 9:e104808.

11. Zhang Q, Jing Q, Gerritsen AE, Witter DJ, Bronkhorst $\mathrm{EM}$, Creugers NH. Dental status of an institutionalized elderly population of 60 years and over in Qingdao, China. Clin Oral Investig 2016; 20(5):1021-1028.

12. Ervin RB, Dye BA. Number of natural and prosthetic teeth impact nutrient intakes of older adults in the United States. Gerodontology 2012; 29(2):e693-702.

13. Hessari H, Vehkalahti MM, Eghbal MJ, Murtomaa H. Tooth loss and prosthodontic rehabilitation among 35to 44-year-old Iranians. J Oral Rehabil 2008; 35(4):245251.

14. Brasil. Ministério da Saúde (MS). Projeto SB Brasil 2010: Pesquisa Nacional de Saúde Bucal 2010. Resultados principais. Brasília: MS; 2011.

15. Roncalli AG, Silva NN, Nascimento AC, Freitas CHSM, Casotti E, Peres KG, Moura Ld, Peres MA, Freire Mdo C, Cortes MI, Vettore MV, Paludetto Júnior M, Figueiredo N, Goes PS, Pinto Rda S, Marques RA, Moysés SJ, Reis SC, Narvai PC. Relevant methodological issues from the SBBrasil 2010 Project for national health surveys. Cad Saúde Pública 2010; 28(Supl.):s40-s57.
16. Adulyanon S, Vourapukjaru J, Sheiham A. Oral impacts affecting daily performance in a low dental disease Thai population. Community Dent Oral Epidemiol 1996; 24(6):385-389.

17. Peres MA, Barbato PR, Reis SC, Freitas CH, Antunes JL. Tooth loss in Brazil: analysis of the 2010 Brazilian Oral Health Survey. Rev Saúde Pública 2013; 47(Supl. 3):78-89.

18. Chalub LL, Ferreira RC, Vargas AM. Functional, esthetical, and periodontal determination of the dentition in 35- to 44-year-old Brazilian adults. Clin Oral Investig 2016; 20(7):1567-1575.

19. Hobdell M, Petersen PE, Clarkson J, Johnson N. Global goals for oral health 2020. Int Dent J 2003; 53(5):285288.

20. Hardcastle WJ. Physiology of speech production. An introduction for speech scientists. London: Academic Press; 1976. p. 136-137.

21. Ichikawa J, Komoda J, Horiuchi M, Matsumoto N. Influence of alterations in the oral environment on speech production. J Oral Rehabil 1995; 22(44):295299.

22. Molly L, Nackaerts O, Vandewiele K, Manders E, van Steenberghe D, Jacobs R. Speech adaptation after treatment of full edentulism through immediate-loaded implant protocols. Clin Oral Implants Res 2008; 19(1):86-90

23. Jacobs R, Manders E, Van Looy C, Lembrechts D, Naert I, van Steenberghe D. Evaluation of speech in patients rehabilitated with various oral implant-supported prostheses. Clin Oral Implants Res 2001; 12(2):167-173.

24. Lundqvist S, Lohmander-Agerskov A, Haraldson T. Speech before and after treatment with bridges on osseointegrated implants in the edentulous upper jaw. Clin Oral Implants Res 1992; 3(2):57-62.

25. Goto T, Nishinaka H, Kashiwabara T, Nagao K, Ichikawa T. Main occluding area in partially edentulous patients: changes Before and after implant treatment. J Oral Rehabil 2012; 39(9):677-683.

26. Martins AMEBL, Jones KM, Souza JGS, Pordeus IA. Associação entre impactos funcionais e psicossociais das desordens bucais e qualidade de vida entre idosos. Cien Saude Colet 2014; 19(8):3461-3478.

27. Martins AMEBL, Barreto SM, Pordeus IA. Factors associated to self perceived need of dental care among Brazilian elderly. Rev Saúde Pública 2008; 42(3):487-496.

28. Freire MC, Reis SC, Figueiredo N, Peres KG, Moreira RS, Antunes JL. Individual and contextual determinants of dental caries in Brazilian 12-year-olds in 2010. Rev Saúde Pública 2013; 47(Supl. 3):40-49.

29. Bhandari B, Newton JT, Bernabé E. Income Inequality and Use of Dental Services in 66 Countries. J Dent Res 2015; 94(8):1048-1054.

Artigo apresentado em 16/08/2016

Aprovado em 03/02/2017

Versão final apresentada em 05/02/2017 
